\title{
Tópicos de políticas de información en el entorno científico y técnico: México 1989 -1994
}

\author{
Miguel Gama \\ Maestro en bibliotecología. \\ E-mail: mgamar@servidor.unam.mx \\ Egbert Vanderkast \\ Maestro en bibliotecología. \\ E-mail: egbert@servidor.unam.mx
}

\section{Resumen}

En primer lugar se hace un recuento sucinto del concierto internacional en materia de políticas de información, luego se reseña la situación de algunos países de América Latina y enseguida se revisa la situación en México con el fin de: a) revisar si el discurso oficial en materia de políticas de información en el campo científico y técnico es considerado en los planes nacionales de desarrollo; b) identificar los tópicos de políticas de información en materia de ciencia y tecnología a partir del análisis de algunas publicaciones oficiales mexicanas y c) profundizar en el periodo 1989 - 1994 que comprende el sexenio del entonces presidente Carlos Salinas de Gortari. La metodología utilizada se refiere al análisis documental de las obras y a la aplicación de una propuesta en particular en torno al estudio de las políticas de información.

\section{Palabras claves}

Políticas de información. México. Ciencia y tecnología.

\section{Topics of information policies in the scientific and technological environment: Mexico 1989-1994}

\section{Abstract \\ In the first place a count becomes resume of the international concert in the matter of information policies, soon the situation of some countries of Latin America is reviewed and immediately the situation in Mexico is reviewed with the purpose of: a) to review if the official speech in the matter of policies of information in the scientific and technical field is considered in the national plans of development; b) to identify the topics of policies of information in the matter of science and technology from the analysis of some Mexican official publications and c) to deepen in the period 1989 - 1994 that includes/understands government of Carlos Salinas de Gortari. The used methodology talks about to the documental analysis of works and to the application of a proposal in individual around the study of the information policies.}

\section{Keywords}

Information policy. México. Science and technology.

\section{INTRODUCCIÓN}

La estructura social y económica de la sociedad se mantiene vigente a través de mecanismos, entre los cuales destacan principalmente: los sistemas que articulan sus acciones a fin de mantener la dinámica social y las políticas públicas definidas por los funcionarios o servidores públicos en turno. Estas engloban las directrices delineadas en cada uno de los elementos de la estructura social, por ejemplo en materias como: educación, ciencia y tecnología, economía, política exterior, entre otras. En su conjunto se les conoce como políticas públicas y desde el punto de vista teórico no deberían sujetarse al corto periodo de una administración, de un gobierno, es decir, a los seis años que por ley duran las gestiones de gobierno para el caso mexicano.

En este contexto es imprescindible la definición de líneas que normen las acciones en torno a administrar esa información y a definir o bien a revisar las políticas públicas que guíen su producción, registro, acceso y por supuesto su preservación.

En la práctica estas directrices están enmarcadas en la visión de los gobiernos, de las entidades, de los servidores públicos y de los organismos de la sociedad civil. En este sentido, las políticas de información en México tienen un expediente voluminoso dentro de la administración pública. Seguramente las líneas generales de esos planteamientos están contenidos en diversas fuentes de información como las que se anotan a continuación: los planes nacionales de desarrollo, los planes sectoriales y los programas e informes de gobierno y de labores. En conjunto representan el eje central que articula todas las acciones en los rubros que integran la administración pública. Sin embargo, de la letra a la realidad existen múltiples elementos que intervienen para el éxito o el fracaso de los objetivos originalmente establecidos. Es por ello relevante llevar a cabo estudios de exploración de la realidad latinoamericana en periodos determinados de su historia.

En este sentido los objetivos del estudio son: a) revisar si el discurso oficial en materia de políticas de información en el campo científico y técnico es considerado en los planes nacionales de desarrollo y b) identificar los tópicos 
de políticas de información en materia de ciencia y tecnología a partir del análisis de algunas publicaciones oficiales mexicanas.

La metodología utilizada se refiere al análisis documental de las obras y a la aplicación de una propuesta en particular en torno al estudio de las políticas de información.

En principio, se hace un recuento sucinto del concierto internacional en materia de políticas de información, luego se reseña la situación de algunos países de América Latina, enseguida se revisa la situación en México y finalmente se profundiza en el periodo 1989 - 1994 que comprende el sexenio del entonces presidente Carlos Salinas de Gortari.

\section{POLÍTICAS DE INFORMACIÓN}

La Unesco, a través de sus diferentes programas, ha sido uno de los entusiastas organismos internacionales preocupados porque cada uno de los países del mundo cuenten con políticas de información y sistemas de información científica y técnica que permitan producir, clasificar, sistematizar, transmitir, difundir, compartir y preservar la información.

De esta manera en la década de los ochenta promovió la realización de seminarios en países de América Latina: Jamaica 1985, Venezuela 1987, México 1987, Colombia 1988, Costa Rica 1988, Uruguay 1989 y Ecuador 1990 con el propósito de analizar las políticas gubernamentales en materia de información.

Además publicó el Manual sobre la Formulación, Aprobación, Aplicación y Funcionamiento de una Política Nacional sobre la Información, en el marco de los trabajos del Programa General de Información (PGI), UNISIST y NATIS, señalando como acciones prioritarias la realización de un estudio preliminar de la situación nacional y la conformación de un organismo encargado de coordinar la ejecución de la estrategia adoptada.

Más tarde, reconoció que una política de información debía considerar aspectos fundamentales con el acceso, el tratamiento y el aprovechamiento de la información.

Definitivamente los primeros pasos dados por la Unesco son fundamentales para el estudio del tema. Al parecer todas las iniciativas emprendidas cristalizaron en una propuesta metodológica para llevar a buen puerto el objetivo trazado: establecer políticas nacionales de información para todos. Cada país tiene su experiencia al respecto, algunos con menor o mayor grado de avance.
Sin embargo, cabe hacerse el cuestionamiento siguiente: ¿Qué debe entenderse por política de información?, para construir la respuesta o las respuestas adecuadas se consultan a los autores que a continuación se señalan: Páez Urdaneta* cita a Gray, experto británico, quien establece que una política nacional de información consiste en determinar cuáles son las finalidades al respecto del país y en velar porque queden satisfechas de un modo tan completo, rápido, poco oneroso y adecuado, como lo permitan unos recursos escasos.

Asimismo el autor enfatiza que una política de información debe contemplar acciones orientadas a la capacitación de recursos, educación de usuarios, introducción de la tecnología de la información y el fomento y apoyo a la realización de investigaciones en el área.

En esta explicación llama la atención el hecho de referirse a política de información en un contexto nacional lo que le da un sentido más amplio respecto a su campo de acción y de influencia.

Sobre el mismo concepto, Carrión Rodríguez ${ }^{* *}$ señala que las políticas de información son decisiones gubernamentales, diseñadas para crear, y/o fortalecer una adecuada infraestructura de información con el fin de impulsar un mayor desarrollo económico, social, cultural e industrial. Reitera que una política de información es un conjunto de principios interrelacionados: leyes, lineamientos, reglas, reglamentaciones e interpretaciones judiciales que guían la vigilancia y administración del ciclo de vida de la información, así como de su acceso y uso. Asimismo destaca que una política de información es una declaración de o de los objetivos específicos que deben lograrse, o deben perseguirse, una declaración de los medios con los que se lograrán dichos objetivos; una reasignación de los medios; y un conjunto de reglas o lineamientos que regulen esta actividad.

Por su parte, la International Encyclopedia of Information and Library Science aclara que no hay un consenso sobre lo que es política de información, pero que ésta es multifacética y puede ser motivada por diversos objetivos los cuales pueden entrar en contradicción. ${ }^{* * * *}$

\footnotetext{
* Cfr. PÁEZ URDANETA, Iraset. Información para el progreso de América Latina. Caracas: Universidad Simón Bolívar. 1990. p.108

*** Cfr. CARRIÓN RODRÍGUEZ, Guadalupe. Diplomado Acceso a la información. Módulo 1. UNAM.DGB, agosto de 1997.

**** Cfr. INTERNATIONAL ENCYCLOPEDIA OF INFORMATION AND LIBRARY SCIENCE, op. cit., p.198.
} 


\section{CUADRO 1}

\section{Políticas de Información}

\begin{tabular}{l|l}
\hline Fuente & Elementos \\
\hline Gray & Identificar Finalidades y Satisfacerlas \\
\hline Carrión Rodríguez & $\begin{array}{l}\text { Decisión Gubernamental, Principios, } \\
\text { Reglas, Lineamientos y Objetivos }\end{array}$ \\
\hline $\begin{array}{l}\text { International Encyclopedia } \\
\text { of Information and }\end{array}$ & Multifacética y diversos objetivos \\
\hline Ponjuán Dante & Qué hacer y para qué \\
\hline
\end{tabular}

Mientras tanto, Ponjuán Dante marca las diferencias entre "política" y "plan" indicando que una política dice qué tipo de sociedad debe existir; un plan sólo dice lo que hará durante un tiempo para lograr ese objetivo. La política apunta hacia la esencia; el plan hacia las actividades. La política tiene que ver con el qué y el por qué; el plan con el cómo.*

El cuadro 1 muestra las ideas vertidas hasta el momento.

De esta forma, el enunciado de Gray se enfoca más al terreno de la exploración a fin de tener presente que se quiere y cómo se hace para conseguirlo. El punto de vista de Carrión Rodríguez se ubica en el escenario público de la política pública para establecer pautas y direcciones a seguir. La orientación de la Enciclopedia es demasiado ambigua en virtud de no señalar tópicos específicos.

La aportación de Ponjuán Dante toca un elemento principal de la política qué hacer y para qué. Dado lo anterior se estima conveniente retomar lo planteado por Carrión Rodríguez en virtud de que ubica el término en el quehacer gubernamental y en una infraestructura de información que tiene impacto en el desarrollo del país como tal y en el desglose de elementos indispensables en todo ciclo de la información para este estudio.

En resumen, se interpreta que la política de información debe constituir la brújula del Estado para que a través de sus representantes definan las líneas a seguir en el terreno de la administración pública y los planes de desarrollo, por su parte, deben establecer los programas y los proyectos en esta materia en el contexto de un periodo determinado.

\footnotetext{
* Cfr. PONJUÁN DANTE, Gloria. "Las políticas nacionales de información en el contexto latinoamericano. El caso de Cuba". En: Ciencias de la Información. Vol. 24 No. 1, marzo 1993. p.44
}

\section{EL PANORAMA LATINOAMERICANO}

En la década de los noventa Molino* en su estudio sobre las Políticas de información en América Latina y el Caribe revela el panorama siguiente: con el propósito de esquematizar la información contenida en el documento señalado se agrupa en varios rubros: primero, el conjunto de disposiciones normativas; segundo, la relación de las instituciones públicas creadas y un tercero, denominado dificultades y fortalezas cuando el autor así lo incluyera. Se enlistan en orden alfabético de acuerdo al nombre del país.

\section{Colombia}

En el aspecto de legislación, la número 29 del 27 de febrero de 1990 establece en su artículo segundo el papel del Estado para organizar un sistema nacional de información científica y tecnológica.

En el mes de julio de 1991 se promulga la nueva Constitución, la cual considera el derecho a la información y se incorporan elementos relativos al acceso a la información.

La nueva ley de política cultural de 1991 anota mecanismos para suministrar información a través de programas de lectura y divulgación.

Respecto a las instancias públicas destacan:

1. Fondo Colombiano de Investigaciones Científicas y Proyectos Especiales, COLCIENCIAS

\section{Consejo Nacional de Ciencia y Tecnología}

3. Instituto Colombiano para el Fomento de la Educación Superior, ICFES

\section{Archivo General de la Nación}

5. Biblioteca Luis Angel Arango

6. Escuela Interamericana de Bibliotecología de la Universidad de Antioquia

Dificultades:**

- Necesidad de garantizar el acceso a la información

- Necesidad de mayor conciencia del valor de la información

\footnotetext{
* Cfr. MOLINO, Enzo. Políticas de información en América Latina y el Caribe. Estudio en siete países de la región. Documento final, mayo $1992.54 \mathrm{p}$.

** Es el término utilizado por Enzo Molino Ravetto en el estudio referido. Sin embargo, se estima que éstas pueden ser consideradas también como debilidades.
} 
- Limitaciones de la infraestructura

- Necesidad de recursos

- Mecanismos de coordinación adecuados

- Capacitación en el uso y manejo de la información

- Preservación del patrimonio informativo nacional

- Desarrollo de un marco normativo

- Articulación entre la política nacional de desarrollo y la de ciencia y tecnología

- Mejoramiento de la calidad y oportunidad de la información

Fortalezas:

- Mayor capacidad del sistema educativo y programas de formación especializada

- Fortalecimiento de la industria editorial, los hábitos de lectura y de los medios de comunicación

- Infraestructura de instituciones de apoyo y de investigación, y grupos de profesionales de la información

- Desarrollo de la industria de la información y de capacidad nacional en informática y telecomunicaciones

- Existencia de recursos de información, servicios referenciales y capacidad de formulación y negociación de proyectos

- Tendencia hacia la descentralización

\section{Costa Rica}

En el aspecto de legislación se encuentra la Ley No. 7169 de Promoción del Desarrollo Científico y Tecnológico del 13 de junio de 1990.

Respecto a las instancias públicas destacan:

1. Consejo Nacional de Investigaciones Científicas y Tecnológicas (CONICIT)

2. Ministerio de Ciencia y Tecnología

3. Consejo Nacional de Rectores

4. Empresa Radiográfica Costarricense (privada)

5. Comisión de Política Informática

Dificultades:

- Necesidad de formación de recursos humanos
- Normalización de procedimientos, software, herramientas, etc.

- Mejor conocimiento de las necesidades de los usuarios y formación de los mismos

- Mecanismos para cobro de los servicios

- Continuidad de los servicios al concluir los apoyos internacionales

- Necesidad de políticas coordinadas de adquisición

- Simplificación de trámites aduanales y para obtención de divisas

- Necesidad de mantener y promover relaciones con organismos de apoyo

- Necesidad de asesoría técnica sobre orientaciones para adquisición de bienes y servicios informáticos

- Limitados volúmenes de producción de materiales editoriales

Oportunidades: ${ }^{7}$

- Demanda creciente de información

- Existe una adecuada infraestructura de comunicaciones

- Se pretende una participación activa en el flujo bidireccional de información

- Se cuenta con bases de datos y con capacidad para crear nuevas

- Se considera indispensable la automatización de la información

- No se explota en forma exhaustiva la información existente en el país

\section{Ecuador}

En el aspecto de legislación se encuentra:

El Plan Nacional de Desarrollo Económico y Social 1989. 1992 que contempla actividades relacionadas en materia de información.

Respecto a las instancias públicas destacan:

1. Consejo Nacional de Ciencia y Tecnología

2. Centro Nacional de Información Referencial

\footnotetext{
* Se considera que el término adecuado debería ser fortalezas.
} 
3. Consejo Nacional de Universidades y Escuelas Politécnicas, CONUEP

\section{Banco Central de Ecuador}

Limitantes:*

- Necesidad de que se reconozca la importancia del sector

- Necesidad de concertar prioridades

- Preservación y conservación del patrimonio documental histórico

- Desarrollo del marco legal

- Normalización

- Fortalecimiento de infraestructura

- Recursos humanos

- Limitación de equipos de cómputo

- Limitaciones en telecomunicaciones

\section{Jamaica}

En el aspecto de legislación se encuentran:

Books Act (1887)

Jamaica Library Service Act (1949)

Decisión 37/74 del 19 de septiembre establece enfáticamente la política de promover el desarrollo de sistemas de información y el establecimiento de NACOLADS.

Institute of Jamaica Act (1978)

Archives Act (1982)

Statistics Act (1984)

Respecto a las instancias públicas destacan:

1. Ministerio de Información y Cultura

2. National Council on Libraries, Archives and Information Systems (NACOLAIS), antes NACOLADS.

3. Consejo de Investigación Científica

4. Instituto de Planeación

5. Universidades de las Indias Occidentales

* Se estima que el término debilidades sería el más pertinente.
Limitantes:

- Necesidad de que se reconozca la información como recurso y de que su planeación sea a nivel nacional

- Debe integrarse como parte del proceso educativo el uso y la concientización de la información

- Es necesario reforzar las medidas de generación y preservación de la producción nacional, y apoyar la industria editorial

- Asegurar el acceso libre y rápido a la información

- Asegurar disponibilidad suficiente y confiable de los recursos necesarios para las unidades de información

- Incorporar nuevas tecnologías de información y de comunicación

- Mejorar la capacitación y el desarrollo profesional de los especialistas, y mejorar sus salarios

- Dar prioridad a los programas de asistencia técnica en este campo

- Asegurar el apoyo necesario al órgano coordinador

- Promover la participación del sector privado

- Mayor participación de los usuarios

- Mejorar los servicios en ciencia y tecnología

- Mejorar el aprovechamiento de la información gubernamental

- Estandarización y control de calidad de la información estadística

- Depósito legal obligatorio

- Simplificación de trámites y reducción de costos de importación de materiales

- Asegurar la relevancia de la información para los usuarios

\section{México}

En el aspecto de legislación no se precisa un ordenamiento en particular.

Respecto a las instancias públicas destacan:

1. Consejo Nacional de Ciencia y Tecnología, CONACYT

2. Universidad Nacional Autónoma de México, UNAM

3. Secretaría de Educación Pública, SEP

4. Asociación Nacional de Universidades e Instituciones de Educación Superior, ANUIES 


\section{Archivo General de la Nación, AGN}

6. Instituto Nacional de Estadística, Geografía e Informática, INEGI

\section{Secretaría de Salud, SSA}

\section{INFOTEC}

\section{Universidad de Colima}

En torno a este país el estudio de Molino Ravetto no señala debilidades, limitaciones o fortalezas.

\section{Uruguay}

En el aspecto de legislación no se precisa un ordenamiento en particular.

Respecto a las instancias públicas destacan:

1. Ministerio de Educación y Cultura

2. Consejo del Sistema Nacional de Información

3. Consejo Nacional de Investigaciones Científicas y Técnicas (CONICYT)

4. Escuela Universitaria de Bibliotecología

5. Asociación de Bibliotecólogos de Uruguay

6. Red Pública de Transmisión de Datos

7. Laboratorios Tecnológicos

Para el caso de Uruguay, el autor tampoco señala debilidades, limitaciones o fortalezas.

\section{Venezuela}

En el aspecto de legislación no se precisa un ordenamiento en particular.

Respecto a las instancias públicas destacan:

1. Instituto Autónomo Biblioteca Nacional

2. Consejo Nacional de Fomento del Libro y la Lectura

3. Banco del Libro

4. Consejo Nacional de Investigaciones Científicas y Tecnológicas (CONICIT)

5. Oficina Central de Informática

6. Archivo General de la Nación

7. Oficina Nacional de Cartografía

8. Colegio de Bibliotecólogos
El apartado de Venezuela no incluye debilidades, limitaciones o fortalezas.

En síntesis, el estudio efectuado por Molino Ravetto muestra que estos siete países de América Latina y el Caribe tienen contrastes significativos en materia de legislación. Es decir, la generación de leyes se ha presentado en forma desigual en el continente. Sólo se anotan disposiciones para cuatro países y para los tres restantes no se incluyen referencias, lo cual en principio podría inferirse como inexistencia de disposiciones legales. Sin embargo, este hecho no necesariamente puede interpretarse como vacíos jurídicos sobre la materia en ese momento. Más bien se estima como una omisión involuntaria por parte del autor del estudio por lo menos para el caso de México.

En este orden de ideas, se observa que Jamaica es uno de los primeros países en tener leyes que se remontan a 1887 lo que implica una añeja experiencia en la materia debido seguramente a su tradición sajona.

En cuanto al contenido de las leyes, principalmente se refiere al campo de la información científica y técnica. Cabe señalar que también se abordan temáticas como: derecho a la información y cultura.

Por otra parte, el estudio de Molino Ravetto revela también que en esta región del mundo las instituciones tienen una presencia significativa ya que en los 7 países señalados se identifica la creación de un conjunto de instancias públicas estrechamente ligadas a la Ciencia y Tecnología, a la Información, a la Educación, a las Bibliotecas y a la Industria, entre otros. En este sentido, en todos los países existe un organismo, que independientemente de la denominación: CONACYT, CONICIT, CONICYT o Consejo de Investigación Científica, impulsa programas y proyectos y que a su vez forman parte de los planes nacionales del gobierno o de desarrollo, según sea el caso.

Mientras tanto, en estos años de la historia de América Latina se aprecia una figura del Estado robustecida en su calidad de promotor de la Ciencia y la Tecnología y como organizador de los sistemas nacionales de información. En este entorno el Estado representa el motor de la acción gubernamental en la mayoría de los sectores y las diversas disposiciones legislativas al respecto fundamentan dicho papel.

Asimismo en el espectro latinoamericano se hace patente la participación de otras entidades de la vida 
pública, económica y social, así como de la iniciativa privada de los países. De esta manera bancos, universidades, archivos, bibliotecas, empresas, institutos, entre otros organismos, han apoyado de una u otra manera las políticas de información en la región.

En torno a los rubros de información, nuevamente se destaca su presencia en los planes nacionales de desarrollo, como por ejemplo en Ecuador, como lo sugieren las recomendaciones de la UNESCO en materia de políticas de información.

Esta particularidad es de vital importancia en el contexto del presente estudio porque es uno de los ejes principales a considerar en el análisis de la realidad mexicana. En la medida que estos planteamientos formen parte de los documentos base del gobierno permitirán al investigador, al estudioso, al interesado en la materia a identificar el rol que ocupa el tema en la concepción, en la mente de los tomadores de decisión o servidores públicos en turno y la forma en la que abordan u orientan las acciones en la práctica dentro de un periodo establecido de la administración pública latinoamericana.

En las últimas décadas del siglo XX y en los primeros años del siglo XXI las actividades en torno a la información, las bibliotecas, los libros, la lectura, las instituciones y las políticas de información siguen siendo temas de interés para amplios sectores de la sociedad en su calidad de actores del entorno: bibliotecólogos, servidores públicos, organizaciones nacionales, así como también para los organismos internacionales.

Morales Campos*, al hacer un recuento de las políticas de información en América Latina destaca lo siguiente:

En Argentina en 1995 se trabaja en un anteproyecto de ley sobre "La creación del Sistema Federal de Servicios de Bibliotecas e Información”. En el mismo sentido se hacen esfuerzos para lograr la promulgación de la Ley de Fomento del Libro y la Lectura.

En Colombia el Plan Nacional de Cultura 1992-1994 considera la creación del Sistema Nacional de Información en el área.

Costa Rica decreta la creación de la Red Nacional de Investigación y Desarrollo en 1992 con el fin de promover los enlaces entre las comunidades nacionales con grupos de especialistas en todo el mundo.

* Cfr. MORAlES CAMPOS, Estela. El derecho a la información y las políticas de información en América Latina. 65th IFLA Council and General Conference, Bangkok, Thailand, August 20-28, 1999. 10 p.
Chile elabora su Ley de Fomento del Libro y la Lectura a partir de 1993. De igual forma dispone de normas legales que protegen los programas de computación y la propiedad intelectual.

México establece en su Constitución Política de los Estados Unidos Mexicanos el derecho a la información, artículo 6 y la libertad de expresión, artículo 7. Además existen ordenamientos relacionados con bibliotecas, derecho de autor y el libro: Ley General de Bibliotecas (1988), Ley Federal de Derecho de Autor (1997) y Ley del Libro.

Perú cuenta con el Sistema Nacional de Bibliotecas a cargo de la Biblioteca Nacional con el propósito de formular y ejecutar políticas en tópicos como: información, bibliotecas, libros y lectura.

Venezuela desempeña acciones a través del Instituto Autónomo Biblioteca Nacional y de Servicios de Bibliotecas, el Banco del Libro y los sistemas y redes de información que existen en los diferentes sectores del país.

El recuento elaborado por Morales Campos esboza múltiples caminos trazados, en su mayoría, por el transitar de los países en casi cinco décadas de experiencias en materia de políticas, información, bibliotecas y libros, entre otros.

El documento reseñado hace énfasis en los ordenamientos jurídicos que regulan estas actividades y aunque es una tarea permanente en esos países, se considera que en algunos casos, como el de México faltaría la actualización de las leyes a fin de incorporar las nuevas necesidades de información en los diversos sectores de la administración pública.

Se observa que la instrumentación de leyes y reglamentos sigue siendo prioridad en los 7 países anotados y que en conjunto comparten intereses y preocupaciones en materia de bibliotecas, información, libros, lectura, derechos de autor y redes, principalmente.

La década de los noventa en América Latina refleja la necesidad de establecer "Sistemas Nacionales" que integren bibliotecas, centros de investigación e información de los diversos sectores sociales, entre otros.

Recientemente, Morales Campos reitera que "en las políticas de información implantadas tanto en México como en América Latina se considera que el ejercicio real de la democracia y de la libertad significa que cada ciudadano tenga 
la oportunidad de informarse, leer, comunicarse, discutir y participar, para motivar la creatividad, el conocimiento científico y el progreso tecnológico, con el fin de modificar comportamientos y elevar niveles de conceptualización teórica".*

Planteamiento que nos permite reflexionar sobre la importancia de la información en el conjunto de la sociedad, el acceso a la misma y la repercusión de ésta en la calidad de vida de los ciudadanos.

\section{LAS POLÍTICAS DE INFORMACIÓN EN EL ENTORNO CIENTÍFICO Y TÉCNICO EN MÉXICO}

En México el Consejo Nacional de Ciencia y Tecnología (CONACYT) fue creado oficialmente en 1970 y ya desde entonces se establecían las funciones siguientes:

“... investigar en forma directa exclusivamente sobre la investigación misma para lo cual deberá especialmente:

a) mejorar y actualizar periódicamente el inventario de recursos humanos, materiales y financieros destinados a la investigación científica y tecnológica,

b) captar o jerarquizar las necesidades nacionales de ciencia y tecnología, estudiar los problemas que las afectan y sus relaciones con la actividad general del país y

c) establecer un servicio nacional de información y documentación científica." ***

En particular, este último inciso es de llamar la atención porque desde esa fecha se delineaba una intención gubernamental en el sentido de proveer una estructura a nivel nacional en torno a la información y documentación científica. Cabe señalar que esta idea prevalecerá, de una u otra forma, en las administraciones gubernamentales futuras del país pero sin llegar a consolidarse hasta nuestros días.

Fernández de la Garza (1973) ${ }^{* * * *}$, en su calidad de Director del Centro de Servicios de Información y Documentación del CONACYT, afirmaba que el objetivo global del Sistema Nacional de Información y Documentación era el proporcionar a todos los usuarios

\footnotetext{
* MORAlES CAMPOS, Estela. La diversidad informativa latinoamericana en México. México: UNAM. Centro Coordinador y Difusor de Estudios Latinoamericanos, 2001. p.302.

** Diario Oficial de la Federación 29 de diciembre de 1970. Ley que crea el Consejo Nacional de Ciencia y Tecnología.

**** Cfr. Fernández de la Garza Guillermo. El Servicio Nacional de Información y Documentación. En Seminario sobre Planeación Arquitectónica de Bibliotecas. Atlihuetzia, Tlaxcala, junio 1973. 11p.
}

la información que necesitaban como apoyo al desarrollo científico y tecnológico del país. Asimismo reiteraba que este servicio era descentralizado en cuanto a la localización física de acervos bibliográficos, personal y servicios, coordinado y fomentado centralmente por el Consejo.

Dicho sistema pretendía acciones particulares en tópicos como: elaborar catálogos colectivos de publicaciones periódicas, obras de consulta y tesis; facilitar la comunicación interbibliotecaria, promover la catalogación centralizada; elaborar diagnósticos de los servicios de información; publicar directorios; crear una infraestructura regional de los enlaces con el SNID; mejorar las bibliotecas en las instituciones de enseñanza superior del país, en especial las de los estados; elaborar listas de acervos básicos para instituciones de enseñanza superior; promover la disponibilidad de recursos financieros a través de organismos gubernamentales y privados; capacitar personal; desarrollar actividades promocionales del uso de los servicios de información; desarrollar un programa para la creación de bibliotecas públicas y fortalecimiento de las existentes, entre otras.

De esta manera el proyecto del Sistema Nacional de Información y Documentación cubría los tópicos siguientes:

- nivel nacional

- infraestructura regional de servicios

- bibliotecas universitarias

- bibliotecas públicas

- acervos documentales

- fuentes de referencia

- cooperación bibliotecaria

- elaboración de diagnósticos

- capacitación

En la década de los ochentas en el marco de las Jornadas Mexicanas de Biblioteconomía realizadas en el estado de Puebla, en la república mexicana. Enzo Molino presentó el trabajo titulado "Avances en el desarrollo del Sistema de Información Científica y Tecnológica en México".

El entonces Director de Servicios Informáticos del CONACYT anotó que el objetivo general del SICYT era el facilitar el acceso eficiente y oportuno de la información científica y tecnológica a todos los interesados. Respecto a su función añadió que era la de 
proveer los servicios de información que requirieran sus usuarios, y en general, podían incluir: suministro de documentos, préstamo de materiales, búsquedas bibliográficas y asesoría en la localización de información, entre otros.

El SICYT contemplaba los servicios siguientes:

a) Servicios bibliográficos (catalogación compartida, canje y donación y adquisiciones)

b) Consulta a bancos de información

c) Referencia

d) Servicios de apoyo documental

e) Programas Generales (capacitación, redes regionales, etc.)

f) Coordinación, apoyos y promoción

Como se puede observar en las líneas anteriores en un lapso de 15 años el CONACYT afinó su percepción en torno a la información científica y técnica, en principio se hizo mención a un Sistema Nacional de Información y Documentación (SNID) y después cambió la denominación a un Sistema de Información Científica y Tecnológica (SICT). Ambas iniciativas contemplaron en esencia tópicos semejantes, destacando el de los bancos de información como novedad en los ochenta.

Hoy día el CONACYT, a 35 años de su creación, ha cambiado y las condiciones sociales y económicas de México también se han transformado. Sin embargo, pese a que existen nuevos entornos y nuevos actores políticos y sociales se considera que la idea de un Sistema Nacional de Información Científica y Técnica de México sigue siendo una propuesta pendiente para el país.

Por ejemplo, la nueva ley del CONACYT, publicada en el Diario Oficial de la Federación del 5 de junio de 2002, hace alusión a un Sistema Integrado de Información sobre Investigación Científica y Técnica.

Dado lo anterior sería conveniente revisar, estudiar y analizar que similitudes o diferencias hay entre esta propuesta contemporánea de sistema de información con aquella que se proponía en los años setenta. Independientemente de ello, la permanencia de este tópico en la agenda gubernamental a través de los años permite sostener que es un tópico relevante para la ciencia y tecnología y para el desarrollo del país.

\section{LAS PUBLICACIONES OFICIALES EN MÉXICO}

En el país las publicaciones oficiales constituyen fuentes de información de primera mano para conocer la actividad de las dependencias públicas. Es por ello importante destacar su importancia en el contexto del proceso de investigación como el que ahora se detalla. Uno de los estudios más reconocidos sobre publicaciones oficiales, es el de Rosa María Fernández* que años después nos aportaría también otra obra en este sentido* y quien sostiene que éstas ofrecen una visión del desarrollo social, económico, educativo, político y cultural del país.

Las publicaciones oficiales pertinentes para el análisis del tema de la presente investigación son:

- Plan Nacional de Desarrollo

- Programa Nacional de Ciencia y Tecnología***

- Informes de Gobierno

- Informes de Gobierno Anexos

- Informes de Ejecución

En su conjunto las obras anotadas contienen las ideas, las actividades, los proyectos prioritarios de la administración en turno y las acciones emprendidas en las múltiples esferas del gobierno federal. Así como de manera particular las referencias a los tópicos de las políticas de información en el entorno científico y técnico.

\section{LOS ESTUDIOS DE LAS POLÍTICAS DE INFORMACIÓN}

Los estudios de las políticas de información en materia de información científica y técnica en México pueden realizarse desde distintas ópticas, visiones, fases de instrumentación o impacto en la sociedad.

A efecto de llevar a cabo el análisis señalado es necesario el uso de esquemas o instrumentos que permitan representar las situaciones de manera práctica. Al respecto existen diferentes puntos de vista y propuestas de estudiosos de las políticas de información, por ejemplo, Rowlands ${ }^{* * * *}$ anota que el estudio de la estructura se basa

\footnotetext{
* Cfr. Fernández Esquivel, Rosa María. Las publicaciones oficiales de México. Guía de publicaciones periódicas y seriadas 1937-1967. México: s.e, 1967, 269 p.

* Cfr. Fernández de Zamora, Rosa María. La gestión editorial del gobierno federal 1970-1993. México: UNAM. CUIB., 165 p.

${ }_{* * *}^{*}$ La denominación de éste puede variar según los criterios que prevalezcan en el gobierno en turno.

****** Cfr. Rowlands, Ian. "Understanding information policy: concepts, frameworks and research tools". En: Journal of Information Science. Vol. 22 No.1, 1996. p.15.
} 
en 3 niveles definidos como políticas transversales, políticas horizontales y políticas verticales.

Las primeras consideradas de manera general con influencia directa e indirecta en el sector de la información; las segundas, dimensionadas a una aplicación específica cuya incidencia se relaciona con el sector de la información y; las terceras, entendidas como aquéllas que tienen una aplicación específica en un sector particular de la información.

De tal manera que la propuesta de Rowlands se resume de la siguiente manera:

\section{CUADRO 2}

Categorización de políticas (Rowlands)

\begin{tabular}{l|l}
\hline Políticas & Sector de la Información \\
\hline Transversales & Influencia directa e indirecta \\
\hline Horizontales & Aplicación específica \\
\hline Verticales & $\begin{array}{l}\text { Aplicación específica en sector } \\
\text { particular }\end{array}$ \\
\hline
\end{tabular}

Por su parte, Anderson* determina el estudio de las políticas en 3 niveles: micropolíticas, políticas de subsistema y macropolíticas. Consideradas las primeras como el conjunto de esfuerzos de los individuos, de las compañías y de las comunidades en asegurar la acción gubernamental favorable para sí mismos; las segundas se centran en sectores de actividad funcionales tales como el control de la contaminación y la regulación de la seguridad en la mina del carbón, las cuales implican lazos entre los comités del congreso, las agencias administrativas y los grupos de interés; las terceras concurren cuando la comunidad y los líderes del gobierno traen a la discusión y la determinación de la política pública.

De esta manera la propuesta de Anderson se refiere a:

\section{CUADRO 3}

\section{Categorización de políticas (Anderson)}

\begin{tabular}{l|l}
\hline Políticas & Entorno \\
\hline Micropolíticas & Esfuerzos individuales y colectivos \\
\hline Subsistema & Acciones en sector de actividad \\
\hline Macropolíticas & Ciudadanos y gobierno \\
\hline
\end{tabular}

* Cfr. Anderson, James E. Public Policy Making. New York: Holt, Rinehart and Winston, Inc. 1984. p.38-42.
Como puede observarse en los cuadros 2 y 3 los autores consultados señalan una matriz conformada por 3 niveles para el análisis de las políticas públicas en materia de información lo que puede interpretarse como una propuesta de modelo conveniente para estudiar y explicar los tópicos en dicho entorno.

Para efectos del presente estudio se utiliza como herramienta de trabajo la propuesta de Rowlands porque su planteamiento está circunscrito al sector de la información y dado que el rubro de la información científica y técnica es el eje central del proyecto se considera el más apropiado para tratar de explicar la realidad mexicana en un periodo de su historia reciente.

Por lo anteriormente expuesto se observó que el Plan Nacional de Desarrollo 1989 - 1994 estableció líneas de acción en los sectores siguientes:

\section{- Telecomunicaciones}

Rectoría del Estado

Expansión de la red básica de telefonía

Desarrollar servicios de transmisión conmutada de datos, de teleinformática, telefonía celular y otros

Modernizar la red de microondas y establecer enlaces troncales de fibra óptica

Contar con un nuevo satélite para 1994

Automatizar la transmisión de telegramas y giros

La lectura del párrafo anterior nos dirige a un sector de la información en particular, el de las Telecomunicaciones, y a partir de éste se estructuran acciones en niveles relacionados con el mismo pero que definitivamente tienen repercusión con el sector de la información. Con base en el esquema de Rowlands lo anterior significaría un tópico de una política horizontal debido a que se refiere a una aplicación específica, las telecomunicaciones, pero que ciertamente tiene impacto en el sector de la información.

Asimismo se percibe un pronunciamiento oficial por mantener la rectoría del Estado en este sector estratégico dentro del contexto de las políticas nacionales de información. En forma explícita el gobierno fijó su posición en esta materia. De tal forma que también cabe la identificación como una política vertical ya que el Estado mexicano se pronuncia sobre un sector en particular, el de las telecomunicaciones, que es un elemento estratégico para el presente y futuro del país.

Por otra parte, el mismo Plan Nacional de Desarrollo anotó otras acciones en materia de: 
- Ciencia y Tecnología

(modernización tecnológica)

"se fortalecerán los acervos de información tecnológica, orientando y estimulando a las instituciones que los administran para que provean este servicio con eficacia a los usuarios de los sectores productivos; y se promoverá a la vez, la función de asistencia técnica y la consultoría de alta calidad de instituciones públicas y privadas, particularmente en beneficio de empresas pequeñas y medianas".*

Al respecto, el apartado de Ciencia y Tecnología se refiere a elementos prioritarios en torno a la información, las unidades de información y las bibliotecas: los acervos y los servicios. Tópicos que forman parte de una política horizontal porque estas directrices están encaminadas a un sector en específico, el de la ciencia y la tecnología y asimismo están circunscritas al rubro de la información.

En relación a otros sectores, el Plan Nacional de Desarrollo señaló:

\section{- Modernizar el Sistema de Salud}

"Consolidar el Sistema Nacional de Información sobre Infraestructura, Programas, Productividad, Control e Impacto de los Servicios de Salud".**

En este sector la publicación oficial estableció una acción en particular sobre un sistema nacional de información que tiene que ver con el área de salud por lo que nuevamente se vislumbra un tópico de una política de información que equivale a una política horizontal de acuerdo a la propuesta de Rowlands que se ha venido utilizando en el análisis del documento.

En este orden de ideas, el Plan Nacional de Desarrollo 1989 - 1994 enfatizó en torno al:

- Aprovechamiento de la Ciencia y Tecnología

"Establecer un sistema de información e intercambio de conocimientos a nivel nacional e internacional sobre ecología, que permita difundir los avances y logros del país en este campo".***

De esta manera el documento rector del gobierno de Salinas de Gortari presentó nuevamente una política

- SECRETARÍA DE PROGRAMACión Y PRESUPUESTO. Plan Nacional de Desarrollo 1989-1994. México: SPP, 1989. p.93.

- Ibidem p.106-107.

... Ibidem p.125. horizontal porque enfatizó una acción en un tema determinado como es la ecología pero que tiene repercusión en materia de información.

En síntesis, en la obra rectora del gobierno mexicano para el periodo 1989 - 1994 se identificaron de manera general temas que configuran tópicos de cuatro políticas horizontales y una política vertical en el contexto de la información científica y técnica (cuadro 4).

\section{CUADRO 4}

Plan Nacional de Desarrollo 1989 - 1994

Tópicos de políticas de información

\begin{tabular}{l|l}
\hline Verticales & Horizontales \\
\hline Rectoría Estado & $\begin{array}{l}\text { 1. Telecomunicaciones } \\
\text { 2. Acervos y Servicios de Información } \\
\text { Tecnológica }\end{array}$ \\
& $\begin{array}{l}\text { 3. Sistema Nacional de Información Salud } \\
\text { 4. Sistema de Información en Ecología }\end{array}$ \\
\hline
\end{tabular}

Por otra parte, el Programa Nacional de Ciencia y Modernización Tecnológica 1990-1994 contiene de manera particular todas aquellas actividades que pretende llevar a cabo el gobierno federal, a través del Consejo Nacional de Ciencia y Tecnología como organismo responsable del área, en el periodo señalado.

El documento se estructura en 7 capítulos: I) Diagnóstico, II) Objetivos, III) Estrategia y Políticas, IV) Financiamiento de la Ciencia y la Modernización Tecnológica, V) Formación de Recursos Humanos, VI) Marco Jurídico y Apoyo Institucional y VII) Lineamientos Generales para la Ejecución del Programa.

La lectura de los mismos nos dirige a las siguientes referencias:

Capítulo I "Con relación a los servicios de consultoría e información tecnológica, cabe destacar que actualmente los bancos de información tecnológica existentes en el país no son de fácil acceso para la mayoría de las empresas; sólo unos cuantos tienen cobertura general; otros se circunscriben a las necesidades de unas cuantas industrias específicas y, por lo común, la búsqueda e interpretación de la información tecnológica requiere personal capacitado, que pueda atender personalmente los requerimientos de las empresas".*

\footnotetext{
* SeCretaría de programación y PRESUPUESTO. Programa Nacional de Ciencia y_Modernización Tecnológica 1990. 1994. México: SPP, 1990. p.9.
} 
Capítulo II "En el caso del sector productivo de la economía, la estrategia de modernización tecnológica reconoce que la responsabilidad fundamental en la decisión de qué tecnologías utilizar corresponde a los productores. El gobierno asegurará las condiciones para que la selección y desarrollo de tecnologías por parte del sector productivo se realice de manera eficiente, eliminando obstáculos y barreras indeseables; confiriendo estabilidad a las políticas de estímulos, financiera, fiscal, comercial, arancelaria, de transferencia de tecnología, de inversión extranjera, etcétera; y facilitando y promoviendo y, en su caso, prestando los servicios de información requeridos".*

Capítulo III "Estimular la captación óptima de ciencia y tecnología generada en el exterior, a través de programas de cooperación e intercambio científico; proyectos conjuntos de investigación; cooperación técnica, científica y cultural; y estudios de posgrado de mexicanos en el exterior; así como aumentando y mejorando la infraestructura de información y servicios de consultoría técnica”.**

En este mismo apartado, se anota "se multiplicarán y fortalecerán los acervos de información tecnológica, orientando y estimulando a las instituciones que los administran para que provean este servicio con eficacia a los usuarios del sector productivo; y se promoverá, a la vez, la función de asistencia técnica y consultoría de alta calidad, particularmente en beneficio de las empresas medianas y pequeñas que no pueden absorber plenamente los costos de la información necesaria para una óptima elección de tecnología".**

En el punto 6.4 del capítulo VI titulado "Infraestructura de Información Científica y Consultoría Técnica” se indican las cuestiones siguientes:

"Crear un sistema de información tecnológica de cobertura nacional, diseñado desde la perspectiva del uso de este tipo de información tecnológica por el sector productivo, que sea manejado por profesionales y que permita el aprovechamiento óptimo de los datos.

Siempre que las disposiciones legales lo permitan, poner al servicio de las empresas, de los centros de

\footnotetext{
${ }^{*}$ Ibidem p.17.

*** Ibidem p.23.

**** Ibidem p. 25 .
}

investigación y desarrollo y de las universidades, las colecciones de patentes registradas en México y las de otros países, impartiendo a los usuarios la capacitación que requieran para su aprovechamiento cabal.

Actualizar los directorios de los programas de investigación y de las publicaciones científicas y tecnológicas disponibles en las universidades y centros de investigación del sector público.

Identificar los mecanismos más modernos y eficaces para la divulgación de información científica y tecnológica.

Realizar y difundir estudios de tendencias de cambio y perfiles tecnológicos por rama industrial”.*

Con base en los párrafos anotados se observa que las acciones del Programa Nacional de Ciencia y Modernización Tecnológica 1990-1994 se concentran principalmente en los rubros siguientes:

- Acervos de información tecnológica (patentes, directorios)

- Bancos de información

- Divulgación de la información científica y tecnológica

- Infraestructura de la información

- Servicios de consultoría técnica

- Servicios de información (modernización tecnológica)

- Sistema nacional de información tecnológica

Estos tópicos coinciden en el área específica de la Ciencia y Tecnología dado que se trata de un programa sobre la materia pero es oportuno señalar que aún muestran un carácter general. Es decir, no se especifica cómo se llevarían a cabo y qué estrategias se instrumentarían al respecto. Su área de acción se ubica, principalmente, en los sectores industrial y empresarial como elementos productivos de la sociedad.

De acuerdo a lo anterior y retomando nuevamente el esquema de Rowlands la representación quedaría de la siguiente forma:

\footnotetext{
* Ibidem p.44.
} 
CUADRO 5

Programa Nacional de Ciencia y Modernización Tecnológica - 1990-1994

Tópicos de políticas de información

\begin{tabular}{l|l}
\hline Verticales & Horizontales \\
\hline & $\begin{array}{l}\text { 1. Acervos y Servicios de Información tecnológica } \\
\text { (Directorios, Publicaciones Científicas y }\end{array}$ \\
& $\begin{array}{l}\text { Tecnológicas, Patentes, entre otros) } \\
\text { 3. Infraestructura de la Información }\end{array}$ \\
& 4. Cansultoría técnica \\
5. Divulgación Científica y Tecnológica
\end{tabular}

El cuadro 5 muestra una política vertical denominada Sistema Nacional de Información Tecnológica, SNIT, porque se entiende como una aplicación específica en un sector particular de la información y 5 rubros de políticas horizontales dirigidas principalmente a: acervos y servicios de información, infraestructura de la misma, consultoría, capacitación y divulgación debido a que se identifican a una aplicación específica pero que tiene repercusión en el sector de la información.

Hasta este momento el esquema utilizado ha permitido esbozar, a partir del discurso gubernamental, un conjunto de tópicos que definitivamente son ingredientes fundamentales de una política de información en materia científica y técnica. De esta manera se infiere que las recomendaciones internacionales en el sentido de que la política de información deberá plasmarse en los contenidos de los planes de desarrollo ha sido cumplida de acuerdo a este primer acercamiento del tema en el expediente mexicano. Sin embargo, hay la certeza de que el análisis deberá complementarse con otras publicaciones oficiales, señaladas anteriormente, para constatar hasta que grado estas directrices se llevaron a la práctica y no solamente quedaron en los contenidos de las publicaciones oficiales mexicanas.

\section{CONSIDERACIONES FINALES}

El trabajo destacó la importancia de las publicaciones oficiales como fuentes de información prioritarias para el análisis de temas de la agenda nacional. Las fuentes consultadas, en esta primera etapa, Plan Nacional de Desarrollo 1989-1994 y Programa Nacional de Ciencia y Modernización Tecnológica 1990-1994 revelaron un conjunto de directrices en materia de información científica y técnica que fueron visualizadas y representadas en el contexto de tópicos de políticas de información de acuerdo a la propuesta de Rowlands.
De tal manera que la política vertical establecida en el Programa Nacional de Ciencia y Modernización Tecnológica 1990 - 1994 (Cuadro 5) denominada Sistema Nacional de Información Tecnológica, SNIT y las 5 políticas horizontales referidas a: acervos y servicios de información tecnológica, infraestructura de la información, consultoría técnica, capacitación y divulgación científica y técnica refrendan el discurso de los tomadores de decisión en torno al tema de políticas de información en el sector científico y técnico.

Así, el entorno de la información científica y técnica visto desde lo que contienen las publicaciones oficiales estableció directrices en tópicos de políticas de información como resultado de un ejercicio gubernamental en particular en México.

Otro aspecto fundamental en este orden de ideas fue el referente a las leyes, normas y disposiciones reguladoras que deberán existir en todos los niveles de la administración pública mexicana con objeto de asegurar la presencia, desarrollo y permanencia del Sistema Nacional de Ciencia y Tecnología y por ende del Sistema de Información Científica y Técnica. Es fundamental que las políticas públicas en la materia estén respaldadas en leyes, reglamentos, acuerdos y disposiciones, principalmente, a fin de evitar interpretaciones por parte de los servidos públicos en turno.

Por último se considera que el análisis reseñado es factible de llevarse a cabo en otros países de América Latina lo cual permitiría conocer en primer instancia si el tema de políticas de información en particular en el terreno de la ciencia y la tecnología forma parte de los programas nacionales de desarrollo y en segundo lugar averiguar cómo se conciben los sistemas de información científica y tecnológica en el continente latinoamericano.

Artigo submetido em 11/03/2006 e aceito em 09/05/2007.

\section{REFERENCIAS}

ANDERSON, James E. Public policy making. New York: Holt, Rinehart and Winston, Inc., 1984. 179 p.

CARRIÓN RODRÍGUEZ, Guadalupe. Diplomado de acceso a la información. [S.1.: s.n.], 1997. Módulo 1. UNAM. DGB, agosto de 1997.

CONSEJO NACIONAL DE CIENCIA Y TECNOLOGÍA. Sistema nacional de información científica y tecnológica: un diseño idealizado tentativo. México: CONACYT: UNAM, 1976.

EMERY, James. C. Sistemas de información para la dirección: el recurso estratégico crítico. Madrid: Ediciones Díaz de los Santos, 1990. 258 p. 
FERNÁNDEZ DE LA GARZA, Guillermo. El servicio nacional de información y documentación. In: SEMINARIO SOBRE PLANEACIÓN ARQUITECTÓNICA DE BIBLIOTECAS, 1973, Atlihuetzia. Anales... Atlihuetzia, Tlaxcala: [s.n.], 1973. 11 p.

FERNÁNDEZ ESQUIVEL, Rosa María. Las publicaciones oficiales de México: guía de publicaciones periódicas y seriadas 1937-1967. México: [s.n.], 1967. 269 p.

. La gestión editorial del gobierno federal 1970-1993. México: UNAM: CUIB, [199-]. 165 p.

INTERNATIONAL encyclopedia of information and library science. London: Routledge, 1997. 492 p.

LASSWELL, Harold D. et al. El estudio de las politicas públicas. México: Miguel Angel Porrúa, 2000. 281 p.

LÓPEZ PIÑERO, J. M.; TERRADA, M. L. La información científica y técnica. Valencia: Instituto de Estudios Documentales e Históricos sobre la Ciencia, 1993.

MÁRQUEZ, María Teresa. 10 años del Consejo Nacional de Ciencia y Tecnología. México: CONACYT, 1982. 493 p.

MÉXICO. Ley que crea el Consejo Nacional de Ciencia y Tecnología. Diario Oficial de la Federación, 29 de diciembre de 1970.

MOLINO RAVETTO, Enzo. El diseño del sistema nacional de información y documentación científica y técnica. In: CONGRESO INTERAMERICANO DE SISTEMAS E INFORMÁTICA, 2., 1974, México. Anales... México: [s.n.], 1974.

- Metodología para la planeación de sistemas de información científica y tecnológica: algunas consideraciones derivadas del caso mexicano: (versión preliminar). París: UNESCO, 1980. (PGI-80/WS/ $3)$.

- Avances en el desarrollo del sistema de información científica y tecnológica en México. In: JORNADAS MEXICANAS DE BiblioteCONOMÍA, 17., 1986, Puebla, Pue. Memorias... México: Asociación Mexicana de Bibliotecarios, A.C., 1988. p. 259. 268.

Perspectivas del sistema de información científica y tecnológica en México. In: SEMINARIO ABIESI 1985, 1985, San Luis Potosí. La interacción de la biblioteca y la informática: anales... Monterrey: Asociación de Bibliotecarios de Instituciones de Enseñanza Superior e Investigación, 1986. p.185-203.
MORALES CAMPOS, Estela. Políticas nacionales de información en México. In: CONGRESO Y CONFERENCIA DE LA FID, 45.,1990, La Habana, Cuba. Anales... La Habana: [s.n.], 1990. 10 p.

\section{(Comp.). SEMINARIO SOBRE POLÍTICAS}

NACIONALES DE INFORMACIÓN PARA LA INVESTIGACIÓN Y EL DESARROLlO, 1., 1990, México. Anales... México: UNAM: CUIB, 1990.98 p.

. El derecho a la información y las políticas de información en América Latina. In: IFLA COUNCIL AND GENERAL CONFERENCE, 65., 1999, Bangkok, Thailand. Anales... Bangkok: [s.n.], 1999.

MURILLO QUIROGA, Sandro. Un panorama aproximado a la información científica y tecnológica en Bolivia. UMSA Revista de Bibliotecología y Ciencias de la Información, dic. 2002.

PÁEZ URDANETA, Iraset. Información para el progreso de América Latina. Caracas: Universidad Simón Bolívar, 1990. 239 p.

PONJUÁN DANTE, Gloria. Las políticas nacionales de información en el contexto latinoamericano: el caso de Cuba. Ciencias de la información, v. 24, n. 1, p. 43-47,1993.

RAMOS, Rosalba Cruz; ZETTER, Julio. Las políticas de información de la UNESCO y su repercusión en América Latina. Revista Española de Documentación Científica, v. 15, n. 4, p. 341-359, 1992.

ROWLANDS, Ian. Understanding information policy: concepts, frameworks and research tools. Journal of Information Science, v. 22, n. 1, p. 13-25, 1996.

SÁNCHEZ VANDERKAST, Egbert. La dependencia informativa. 1996. 124 f. Tese (Mestrado em Biblioteconomia)- México, 1996.

SECRETARÍA DE PROGRAMACIÓN Y PRESUPUESTO. Plan Nacional de Desarrollo 1989 - 1994. México: SPP, 1989. 143 p.

. Programa nacional de ciencia y modernización tecnológica, 1990-1994. México: SPP, 1990. 48 p.

ZALDÍVAR COLLAZO, Modesto. Importancia de una política nacional de información. Ciencias de la Información, v. 23, n. 1, p. 15. 22, 1992. 